\title{
EASO AWARDS
}

\section{EASO Young Investigator Award: Basic Science}

J. Hoeks

${ }^{1}$ MAASTRICHT UNIVERSITY, Maastricht, Netherlands

Abstract text: Obesity leads to fat accumulation in muscle and liver Abstract text: Obesity leads to fat accumulation in muscle and liver, that acute elevation of circulating fatty acids via lipid infusion increased intramuscular triacylglycerol (IMTG) levels and induced insulin resistance. However, a lipid emulsion based on medium-chain fatty resistance. However, a lipid emulsion based on medium-chain fatty
acids lead to a similar reduction in insulin sensitivity but no IMTG accumulation in skeletal muscle, indicating that muscle IMTG accumulation is not a prerequisite for the induction of insulin resistance. We also showed that lipid infusion significantly reduced the expression of several key genes involved in mitochondrial metabolism. Furthermore, prolonged fasting in healthy lean subjects, leading to highly elevated plasma free fatty acid levels and insulin resistance, significantly reduced mitochondrial respiration in permeabilized muscle fibers. This may suggest that lipid-induced mitochondrial dysfunction is .

Interestingly, it has been shown that lipid-induced insulin resistance and -mitochondrial dysfunction are associated with elevated production of reactive oxygen species (ROS). The protein levels of UCP3, a protein putatively involved in the protection against (lipid-induced) oxidative stress via mitochondrial uncoupling, are reduced to $~ 50 \%$ in both type 2 diabetic and obese subjects with impaired glucose tolerance. However, our studies with UCP3 knockout or overexpressing mice did not reveal differences in mitochondrial uncoupling and ROS production. Furthermore targeting ROS production with a mitochondria-specific antioxidant did not prevent high fat diet-induced insulin resistance and did not improve mitochondrial function. These results do not support a causal role for UCP3 or oxidative stress in lipid-induced insulin resistance and -mitochondrial dysfunction in skeletal muscle. Conflict of Interest: Funding:

\section{EASO Young Investigator Award: Clinical Research}

ADIPOKINE DYSREGULATION AND ADIPOSE TISSUE INFLAMMATION IN HUMAN OBESITY

V. Catalán

CLÍNICA UNIVERSIDAD DE NAVARRA, Metabolic Research Laboratory, Pamplona, Spain

CIBER Fisiopatología de la Obesidad y la Nutrición, ISCIII

Abstract text: Adipose tissue is a complex organ that regulates and coordinates metabolic homeostatic mechanisms. Abdominal obesity is a well-established metabolic risk factor for the development of insulin resistance and type 2 diabetes. Compelling evidence points to the fact that an excess of visceral adipose tissue accumulation dysregulates the adipokine secretion profile, which results in adverse effects on glucose and lipid metabolism. It is also increasingly recognised that obesity is and lipid metabolism. It is also increasingly recognised that obesity is associated to a low-grade chronic inflammation as well as to an increased oxidative stress, both related to the initiation and propagation
of metabolic disorders and representing key etiological factors in linking of metabolic disorders and representing key etiological factors in linking increased adiposity with type 2 diabetes development. Several novel YKL-40, calprotectin, osteopontin, chemerin and proteins of the extracellular matrix. Moreover, the evidence that fat mass accretion is associated with an accumulation of adipose tissue macrophages raises the hypothesis that intlammation is a primary event in the genes the hypothesis that adipocyte development and function, thereby playing an important role in weight regulation, obesity and lipid metabolism. Much attention has focused on adipose tissue changes in the extracellular matrix and inflammation linked to obesity, highlighting the interrelation of both processes in the adipose gene expression levels of obese subjects. Conflict of Interest: Funding:

\section{EASO Young Investigator Award: Public Health}

"MY PARENTS ARE BIG, IT RUNS IN THE FAMILY": INHERITED BEHAVIOURAL SUSCEPTIBILITY TO OBESITY C. Llewellyn

UNIVERSITY COLLEGE LONDON, Health Behaviour Research Centre, Department of Epidemiology and Public Health, London, United Kingdom

Abstract text: Despite the ubiquitous 'obesogenic' environment there is substantial variation in weight. Adiposity is highly heritable (50-90\%) and GWASs have identified a number of common genetic variants. Variation in genetic susceptibility to the environment is thought to partly explain variation in weight, with differential appetitive responses to food being implicated as the mediating mechanism - so-called Inherited Behavioural Susceptibility Theory (IBST). IBST hypothesises that heritable variation in appetite avidity and satiety sensitivity influence the risk of overeating in response to the modern food environment, and consequently gaining excessive weight. Gemini, a large populationcon identify early causal processes in weight gain, with a focus on behavioural pathways. A new psychometric measure of infant appetite (the Baby Eating Behaviour Questionnaire, BEBQ) was developed using the Gemini sample. The BEBQ data demonstrated: i) there are individual differences in appetitive traits in infancy, ii) appetitive traits are prospectively associated with weight gain in early life, iii) appetitive traits are highly heritable in infancy; and iv) associations between appetitive traits and weight have a common genetic pathway. These results provided solid support for IBST. The findings suggest that weight variation in infancy may be due partly to genetically determined differionces in appetitive (if obesogenic ' environments. IBST implies that obesity is not simply a matter of personal responsibility, but an interaction between individual vulnerability and environmental exposure, highlighting the need for greater environmental modification to prevent overeating for susceptible individuals.

Conflict of Interest:

Funding: 